\title{
LED Modulation Characteristics in a Visible-Light Communication System*
}

\author{
Yanrong Pei, Shaoxin Zhu, Hua Yang, Lixia Zhao, Xiaoyan Yi, Junxi Wang, Jinmin Li \\ Institute of Semiconductors, Chinese Academy of Sciences, Beijing, China \\ Email: yrpei@semi.ac.cn
}

Received 2013

\begin{abstract}
This paper conducts a research on modulation characteristics of blue light-emitting diodes (LEDs) used in a visible-light communication (VLC) system. Through analysis of the modulation characteristics of LEDs with different emitting sizes, we find that there is a similar linear relationship between LED's 3dB bandwidth and the operation current density. This experiment also shows that high series resistance is one major issue that limits our LED's modulation speed. To further improve the LED bandwidth, the resistance can be reduced by optimizing device layout as well as reducing material bulk resistance. Clearly, this study provides an approach to increase the modulation bandwidth of GaN-based LEDs for VLC systems.
\end{abstract}

Keywords: LED; Visible-light Communication (VLC); Modulation Characteristics; 3dB Bandwidth; Current Density

\section{Introduction}

With the rapid development of modern solid-state lighting, light-emitting diodes (LEDs) are increasingly used in a wide range of display, signaling and illumination applications [1]. Because of the long lifetime and high energy efficiency, LED is becoming one of the dominant illumination technologies [1,2]. In addition, these small-sized and energy-efficient devices tend to be used in both illumination and communication: visible-light communication (VLC) is attracting a lot of research interests in Asia, Europe and the U.S. [1,3]. Using GaNbased LEDs as the signal sources in VLC systems, the modulated signal is able to transmit digital data beyond the perception speed of human eyes [4]. Therefore, the double functions of LEDs make them popular in recent researches on the area of free space VLC system.

A nonreturn-to-zero (NRZ) system using a postequalized white LED at $100 \mathrm{Mb} / \mathrm{s}$ has been reported in [1], and a discrete multi-tones (DMT) modulation of white LED has reached a data rate of $513 \mathrm{Mbit} / \mathrm{s}$ in [5]. Furthermore, a single RGB LED luminary with the DMT modulation has achieved a rate of $813 \mathrm{Mbit} / \mathrm{s}$ in [6]. A research of white LED modulation bandwidth and modulation characteristics has been reported in [4]. And also the LED spatial light intensity effect on the amplitude-frequency characteristics of VLC has been reported in [7]. Response frequency of LED is directly affecting the avail-

*This work supported by the National High Technology Research and Development Program of China (863 Program), No. 2011AA03A105. able bandwidth of VLC system [4]. Wherefore, increasing the frequency response of LED is the primary event to achieve high speed VLC, when chasing high-power LED simultaneously [4]. In this paper, we demonstrate that the current density on LED affects the response frequency of LED, which determines the modulation bandwidth of LED.

\section{Experimental Setup}

As Figure 1 inset shows that the wavelength of LED in this experiment is approximately $450 \mathrm{~nm}$, which is the common wavelength of blue LEDs. This curve is measured with the integrating sphere using $45 * 45$ mil blue LED.

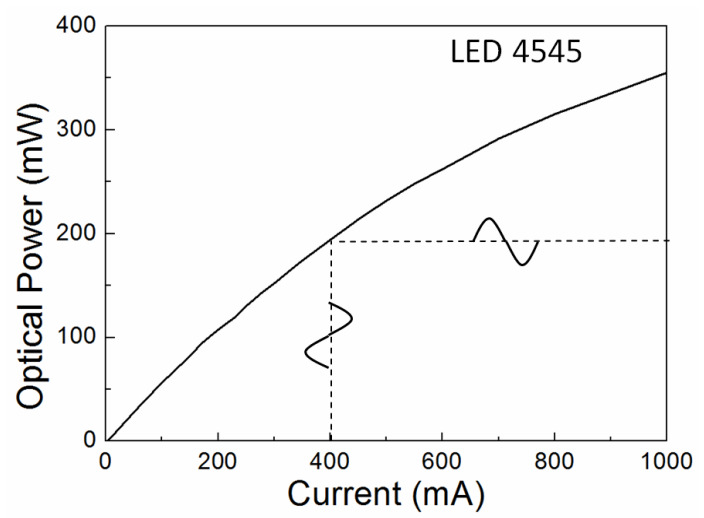

Figure1. The P-I curve of LED modulation. Inset: The spectra of the light sources in this experiment. 
A typical LED's current-voltage (I-V) curve is clearly known. The LED is single conduction and when the bias voltage exceeds a certain turn-on value $\mathrm{V}_{\mathrm{A}}$, the LED can operate in the linear region (work area). The turn-on voltage of the LED in this study is about $2.8 \mathrm{~V}$. Therefore, in order to ensure the LED work in the linear region, the bias voltage should be higher than $3 \mathrm{~V}$. The modulation capability of a LED is described with optical power and electrical current (P-I curve). In Figure1, the P-I curve was approximately linear without a threshold current, so the LED's optical power output can be linearly modulated with a small input voltage signal that is biased above $\mathrm{V}_{\mathrm{A}}$.

The bandwidth measurement setup for the VLC system is shown in Figure 2 and the parameters of each component were listed in Table 1. The VLC transmitter consists of an amplifier a power supply and a bias-T. The receiver comprises a photo-detector that was PositiveIntrinsic Negative diode (PIN diode) or Avalanche Photo Diode (APD). LED that was the light source in transmitter emits visible light and then absorbed by receiver through free space spread. The two-port network analyzer works as a signal source and also a terminal analyzer, providing a small sine wave as a function generator and measuring the received amplitude as well.

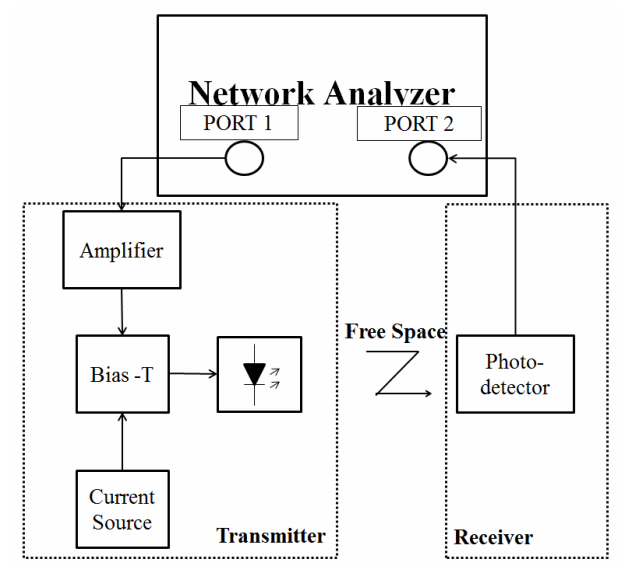

Figure 2. The experimental setup of VLC system.

Table 1. VLC system parameters.

\begin{tabular}{lc}
\hline \multicolumn{1}{c}{ Parameters } & Values \\
\hline $\begin{array}{l}\text { Photo-detector } \\
\text { detection area }\end{array}$ & $0.8 \mathrm{~mm}^{2}$ \\
$\begin{array}{l}\text { Photo-detector } \\
\text { bandwidth }\end{array}$ & $150 \mathrm{MHz}$ \\
\hline Amplifier gain & $25 \mathrm{~dB}$ \\
\hline Amplifier frequency & $500 \mathrm{MHz}$ \\
Bias-T current (max.) & $500 \mathrm{~mA}$ \\
\hline Bias-T frequency & $4200 \mathrm{MHz}$ \\
\hline Load & $50 \Omega$ \\
\hline
\end{tabular}

The modulation bandwidth of LED is restricted with the response frequency. However, the minority carriers' lifetime in semiconductors affect this response frequency. Therefore, the theoretical bandwidth of LED was limited below $2 \mathrm{GHz}$ [8]. Currently, the bandwidth of LED is far below this theoretical value. Hence, the lower modulation bandwidth of LED affects its application in the field of high-speed communications. In the following parts, the research for modulation characteristics of LED based on the above experimental setups of VLC system is described.

Table 1 shows the parameters in the experimental setups of VLC system. These parameters ensure the accuracy and reliability of the experimental results.

\section{Results and Discussion}

In the following parts, the research for modulation characteristics of LED based on the above experimental setups of VLC system is described.

The optical source in this experimental was some different sizes LEDs (blue LED without yellow phosphor). The chip sizes of LEDs A, B and C are $07 * 09$ mil, $10 *$ 23 mil and $45 * 45$ mil, respectively. Or, in other words, the active area of LED A is $0.04 \mathrm{~mm}^{2}$; and the active area of LED B is $0.15 \mathrm{~mm}^{2}$; and lastly, the active area of LED $\mathrm{C}$ is $1.3 \mathrm{~mm}^{2}$.

The three different experimental LEDs were divided into three groups, which were measured under the same light intensity, and then current density became the only variable. Therefore, the results can directly reflect the relationship between current density and the $3 \mathrm{~dB}$ bandwidth of LED. In Figure 3, the Y coordinates, which is $\mathrm{S}_{21}$ Amplitude, means that the ratio between output power and input power and it reflects the $3 \mathrm{~dB}$ bandwidth of LED. Because of the background noise in this experiment, the curves in Figure $\mathbf{3}$ have large fluctuations. However, these non-smooth curves are not affecting the overall trend of the experimental results.

As Figure 3 shows that the $3 \mathrm{~dB}$ bandwidth of LED A was $10.5 \mathrm{MHz}$ in the bias current of $20 \mathrm{~mA}$ and the value rapid increased to $44 \mathrm{MHz}$ with the bias current of $100 \mathrm{~mA}$. In addition, the LED $\mathrm{B}$ and LED $\mathrm{C}$ also demonstrated this rule and the results were shown in Figures 3(b) and (c), respectively. Therefore, the measured $3 \mathrm{~dB}$ bandwidth of three different sizes LEDs were all improved significantly with the increased current density. This phenomenon can be demonstrated with the probability of bimolecular recombination, which was proportional to the injected carrier density into the active volume $[9,10]$.

In Figure 4, it was clear that there was a similar linear relationship between current density and the $3 \mathrm{~dB}$ bandwidth at each size of LEDs, which were $07 * 09$ mil, 10 * 23 mil and $45 * 45$ mil. Although $45 * 45$ mil LED C possesses largest capacitance due to its larger active area, 


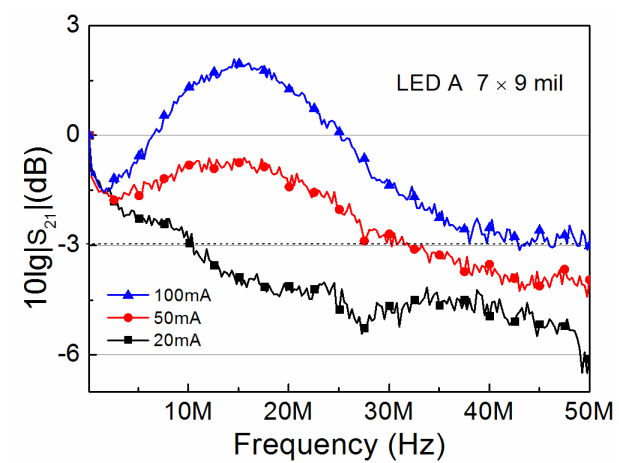

(a)

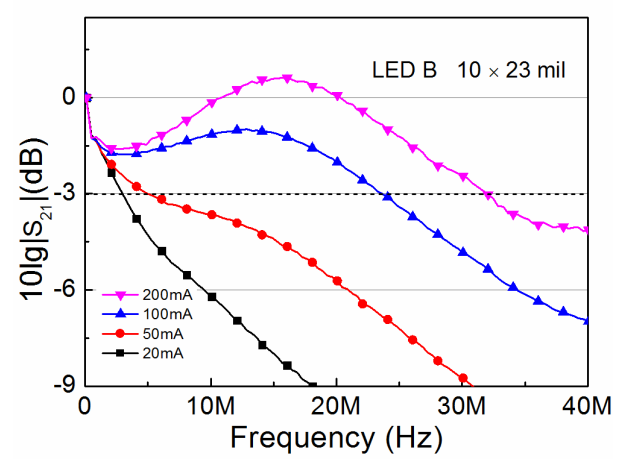

(b)

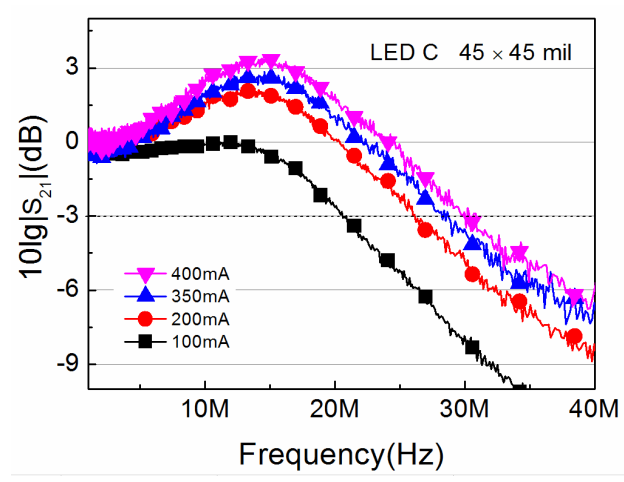

(c)

Figure 3. The normalized frequency response of (a) LED A, (b) LED $B$ and (c) LED C measured under different bias currents.

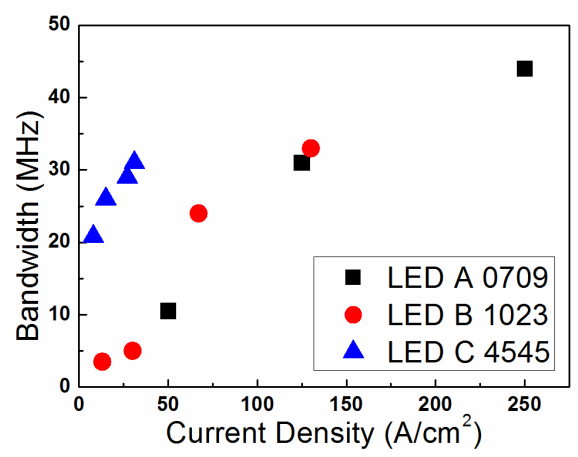

Figure 4. The diagram of the relationship between current density and $3 \mathrm{~dB}$ bandwidth. the smaller resistance, which helps to reduce more parasitic, thus improve the high speed performance at low current density. Therefore, high series resistance is one major issue that limits our LED's modulation speed. To further improve the LED bandwidth, the resistance can be reduced by optimizing device layout as well as reducing material bulk resistance.

These experimental results from the above setups that further evidence the relationship between $3 \mathrm{~dB}$ bandwidth of LED and the current density into LED.

\section{Summary and Conclusions}

In this paper, a measurement setup of modulation characteristics for VLC systems is described and the bandwidths of different-sized blue LEDs have been reported. The results clearly reveal a similar linear relationship between LED current density and its $3 \mathrm{~dB}$ bandwidth. This phenomenon can be attributed to the bimolecular recombination probability that is proportional to the injected carrier density into the active volume. Therefore, increasing the LED current density is a feasible method in VLC systems to enhance the data transmission rate. Moreover, this experiment shows that high series resistance is one major issue that limits our LED's modulation speed. Thus, further study will focus on optimizing device layout as well as reducing material bulk resistance to reduce the resistance.

\section{REFERENCES}

[1] H.L. Minh, D. O’Brien, G. Faulkner, L. Zeng, K. Lee, D. Jung, Y. J. Oh and E. T. Won, " $100-\mathrm{Mb} / \mathrm{s}$ NRZ Visible Light Communications Using a Postequalized White LED" in IEEE Photonics Technology Letters, Vol. 21, No.15, August 2009.

[2] F. L. Jenq, T. J. Liu and F. Y. Leu, "An AC LED Smart Lighting System with Visible Light Time-Division Multiplexing Free Space Optical Communication," 2011 Fifth International Conference on Innovative Mobile and Internet Services in Ubiquitous Computing. doi:10.1109/IMIS.2011.66

[3] Z. W, J. Chau and T. Little, "Modeling and Designing of a New Indoor Free Space Visible Light Communication System," Networks and Optical Communications (NOC) in 16th European Conference, July 2011, pp. 72-75.

[4] J. Cao, Z. Liang and Z. Ma, "White LED Modulation Bandwidth and Modulation Characteristics of the Study" Hans Journal of Wireless Communications, 2012.

[5] J. Vučić, C. Kottke, S. Nerreter, K.-D. Langer and W. Waleski, "513 Mbit/s Visible Light Communication Link Based on DMT-Modulation of a White LED," Journal of Light Technology, Vol. 28, No. 24, 2010.

[6] J. Vučić, C. Kottke, K. Habel and K.-D. Langer, "803 Mbit/s Visible Light WDM Link based on DMT Modulation of a single RGB LED Luminary," Optical Fiber 
Communication Conference and Exposition (OFC/NFOEC), 2011 and the National Fiber Optic Engineers Conference, Conference Publications, March 2011, pp.1-3.

[7] Z. Xu, H. Liang, Z. Zhong, L. He and X. Gu, "Effect of High-Power LED Spatial Light Intensity on Amplitude-Frequency Characteristics of VLC," Optical Communication Technology, Vol. 35, 2011.

[8] R. D. Koudelka and J. M. Woodall, "Light Emitting Devices with Increased Modulation Bandwidth," Yale Uni- versity, 2011.

[9] P. Bhattacharya, "Semiconductor Optoelectronic Device," Pearson Education, 2003, pp. 216-218.

[10] K. Ikeda, S. Horiuchi, T. Tanaka and W. Susaki, "Design Parameters of Frequency Response of GaAs-(Ga,Al) As double Heterostructure LED's for optical communications," IEEE Tansactions on Electron Devices, Vol. ED-24, No. 7, 1977, pp. 1001-1005. doi:10.1109/T-ED.1977.18869 